\title{
AN ANALYSIS OF A SINGULARLY PERTURBED TWO-POINT BOUNDARY VALUE PROBLEM USING ONLY FINITE ELEMENT TECHNIQUES
}

\author{
MARTIN STYNES AND EUGENE O'RIORDAN
}

\begin{abstract}
We give a new analysis of Petrov-Galerkin finite element methods for solving linear singularly perturbed two-point boundary value problems without turning points. No use is made of finite difference methodology such as discrete maximum principles, nor of asymptotic expansions. On meshes which are either arbitrary or slightly restricted, we derive energy norm and $L^{2}$ norm error bounds. These bounds are uniform in the perturbation parameter. Our proof uses a variation on the classical Aubin-Nitsche argument, which is novel insofar as the $L^{2}$ bound is obtained independently of the energy norm bound.
\end{abstract}

\section{INTRODUCTION}

We consider the analysis of finite element methods for the singularly perturbed problem

$$
\begin{gathered}
L u(x) \equiv-\varepsilon u^{\prime \prime}(x)+a(x) u^{\prime}(x)+b(x) u(x)=f(x), \quad 0<x<1, \\
u(0)=u(1)=0,
\end{gathered}
$$

where $\varepsilon \in(0,1]$ is a parameter, $a \in C^{2}[0,1], b \in C^{1}[0,1], f \in C^{1}[0,1]$, and for $x \in[0,1]$ we have

$$
a(x)>\alpha>0 .
$$

We assume that problem (1.1) has a unique solution $u(x)$. This is guaranteed if $\varepsilon$ is sufficiently small (see, e.g., Gartland [4, p. 97]). In general, this solution has a boundary layer at $x=1$. It is possible in our analysis to weaken the differentiability assumptions on $a, b$, and $f$, but for simplicity of presentation we have not done this.

Problem (1.1) may be regarded as a linearized one-dimensional version of a convection-dominated flow problem. Many authors have suggested methods for its numerical solution, and at present it is well understood from a computational point of view. However, the analysis of such methods (i.e., the provision

Received April 4, 1989; revised November 22, 1989.

1980 Mathematics Subject Classification (1985 Revision). Primary 65L10, 65L60; Secondary $34 \mathrm{E} 15$.

The first author's research was partly supported by Arts Faculty Research Fund, University College, Cork. 
of proofs that numerical algorithms yield accurate approximate solutions) is difficult. We shall confine our attention to analyses which yield realistic upper bounds on the errors obtained in actual calculations. This excludes, for example, arguments whose error bounds involve Sobolev norms of $u$, because such norms involve negative powers of the parameter $\varepsilon$, and so the bounds are in general several orders of magnitude greater than the errors obtained in practice.

Since the seminal papers of $\mathrm{Il}^{\prime}$ in [8] and Kellogg and Tsan [9], various numerical methods for the solution of (1.1) have been analyzed using finite difference techniques (see, e.g., Berger, Solomon, and Ciment [2], Emelyanov [3], Gartland [5], and their references). This encompasses papers such as Gartland [4] and Stynes and O'Riordan [15], where finite elements are used to generate difference schemes, but the argument is essentially carried out in the consistency/stability framework associated with finite difference methods. Most analyses consider difference schemes which satisfy a discrete maximum principle; this is a serious restriction if one wishes to generalize these arguments to problems in two dimensions, because there a linear nine-point scheme which is of positive type cannot have truncation error of order greater than one, uniformly in $\varepsilon$ (see Van Veldhuizen [19]). However, we note that the papers of Niederdrenk and Yserentant [12] and Gartland [5] do not employ discrete maximum principles.

In comparison with the finite difference situation, it seems more troublesome to adapt "classical" finite element arguments to yield realistic error bounds for approximate solutions of (1.1). An appreciation of the difficulties involved may be gained by examining Axelsson [1] or de Groen [6]. These difficulties arise because of the asymmetric nature of the bilinear form associated with (1.1). (They manifest themselves, for example, in inequality (3.9) of [6], which gives an upper bound for this bilinear form; this upper bound is clearly not tight when both arguments of the bilinear form equal $u$.)

It is possible to make some progress by constructing a symmetric bilinear form which is equivalent to the original asymmetric one. This gives an elegant theory which measures the effectiveness of any proposed test space (see Morton [10]). In general, however, there are practical difficulties in computing the "ideal" test functions prescribed by the theory, as described in Morton and Scotney [11].

Another approach is that of Szymczak and Babuška [18], where $L^{p}$ estimates are obtained via a finite element analysis; however, the argument used relies on bounds for the Green's function of the differential operator $L$, and so seems difficult to extend to higher-dimensional problems.

In this paper we show how certain Petrov-Galerkin methods for (1.1) may be analyzed using a purely finite element approach and without attempting to symmetrize the associated bilinear form. No use is made of discrete maximum principles nor of equivalent ideas such as nonnegative discrete Green's functions. We need bounds on $\left|u^{(i)}(x)\right|, i=0,1,2$, but no asymptotic expansion of $u$ is required. We work with fairly general meshes which from a practical point of view are essentially arbitrary. We consider several difference schemes 
generated by making various choices for the trial and test spaces. We obtain error bounds in the usual energy norm associated with $(1.1)$ and in the $L^{2}[0,1]$ norm. The magnitude of these error bounds does not depend on $\varepsilon$; in the singular perturbation literature, numerical methods with this property are said to be uniformly accurate or uniformly convergent. It is of some interest to note that our $L^{2}$ estimate is obtained independently of the energy norm estimate; in this way, our analysis is quite different from the classical Aubin-Nitsche approach. Our work sheds light on the relationship between the choice of trial and test spaces and the norm (energy or $L^{2}$ ) in which one can prove a uniform convergence result (see Remark 3.1). The results also illustrate the importance of using a suitable quadrature rule when dealing with singular perturbation problems, as a comparison of $\S \S 4,5$, and 6 reveals.

An outline of the argument we use to obtain our energy norm bound (in the case of a Galerkin finite element method on a uniform mesh) is given in Stynes and O'Riordan [16]. In O' Riordan and Stynes [13] we show that a variation of this argument is valid in the case of a constant-coefficient singularly perturbed elliptic problem in two dimensions, using again a Galerkin finite element method on a uniform mesh. For brevity, several proofs below are either summarized or omitted; in all cases the entire argument can be found in the technical report [17].

It is not the purpose of this paper to suggest new methods for solving (1.1) numerically, as adequate methods already exist, but rather to construct a framework suitable for the finite element analysis of singularly perturbed equations. Consequently, we do not present any numerical results. Nevertheless, we point out that two-dimensional analogues of the finite element methods of this paper can be applied successfully to singularly perturbed elliptic problems, and that numerical results for these will appear in Hegarty, O'Riordan, and Stynes [7].

\section{THE CONTINUOUS PROBLEM}

In this section we discuss those properties of (1.1) and of its solution $u$ which we shall need later for the analysis of our finite element method.

Notation. Throughout this paper we shall use $C$ to denote a generic positive constant which is independent of $\varepsilon$ and of the mesh used. We use $C_{i}$, $i=1,2,3$, to denote specific positive constants (arising in proofs) which are independent of $\varepsilon$ and of the mesh used. We shall say that a quantity $q$ is $O(z)$ when we mean that $|q| \leq C z$ for all sufficiently small $z$.

First we deduce an inequality needed later to show that certain bilinear forms associated with the operator $L$ are coercive.

Lemma 2.1. Without loss of generality, we may assume that there exists $C_{1}>0$ such that for $x \in[0,1]$ we have

$$
b(x)-\frac{1}{2} a^{\prime}(x) \geq 2 C_{1} .
$$


Proof. This can be achieved by a change of variable of the form $v=e^{-\sigma x} u$ with suitably chosen $\sigma$. See [17] for details.

From now on, we shall assume that $(1.1 \mathrm{~d})$ is satisfied in addition to $(1.1 \mathrm{a}-$ c). We also assume, without loss of generality, that $C_{1} \leq 1 / 2$.

Lemma 2.2. The solution $u$ of (1.1) satisfies

$$
\|u\|_{L^{\infty}[0,1]} \equiv\|u\|_{\infty} \leq C
$$

and

$$
\left|u^{(i)}(x)\right| \leq C\left(1+\varepsilon^{-i} e^{-\alpha(1-x) / \varepsilon}\right) \text { for } 0<x<1 \text { and } i=1,2 .
$$

Proof. These bounds are immediate from Kellogg and Tsan [9] when $b \geq 0$. If we do not have $b \geq 0$, then proceed analogously to the proof of Lemma 2.1 .

Our analysis will make repeated use of the arithmetic-geometric mean inequality

$$
y z \leq t y^{2}+z^{2} / 4 t, \quad t>0
$$

\section{Petrov-Galerkin finite element discretizations}

To begin with, we work with an arbitrary mesh

$$
0=x_{0}<x_{1}<\cdots<x_{N}=1,
$$

and we set $h_{i}=x_{i}-x_{i-1}$ for $i=1, \ldots, N$, with $H=\max _{i} h_{i}$. For $i=$ $1, \ldots, N-1$, set $\tilde{h}_{i}=\left(h_{i}+h_{i+1}\right) / 2$. The energy norm estimate of $\S 4$ is valid on this mesh; in $\S \S 5$ and 6 we shall consider a slightly less general mesh which is described in $\S 5$.

We assume that $\varepsilon$ is so small that $(2 \varepsilon / \alpha) \ln (1 / \varepsilon)<1 / 2$. Set

$$
M=\max \left\{i: x_{i} \leq 1-(2 \varepsilon / \alpha) \ln (1 / \varepsilon)\right\} .
$$

From (2.2) we have

$$
\left|u^{\prime}\right|,\left|u^{\prime \prime}\right| \leq C \text { on }\left(0, x_{M}\right) .
$$

We shall refer to $\left[x_{M}, 1\right]$ as the layer region. Outside $\left[x_{M}, 1\right]$, we use piecewise linear "hat" functions in both the trial and test spaces; as Axelsson [1] has pointed out, one needs to use some form of exponential upwinding only in the neighborhood of $x=1$. In the layer region $\left[x_{M}, 1\right]$ we consider three possibilities:

(i) using approximate $L$-spline trial functions and hat test functions ( $(4)$,

(ii) using hat trial functions and approximate $L^{*}$-spline test functions $(\S 5)$,

(iii) using approximate $L$-spline trials and approximate $L^{*}$-spline tests $(\S 6)$. 
In all cases, the integrals used to define the schemes can be evaluated exactly. In general, the difference schemes generated will not satisfy a discrete maximum principle.

Remark 3.1. Possibility (i) above will yield an energy norm result, possibility (ii) yields our $L^{2}$ norm result, while (iii) yields the same $L^{2}$ norm result and an improved energy norm result. This trichotomy illustrates the role played by each space in obtaining uniformly accurate numerical methods for singular perturbation problems.

\section{4. $\widetilde{L}$-SPLINE TRIAL FUNCTIONS IN THE LAYER REGION}

In this secton we give a Petrov-Galerkin finite element method for which we derive an error estimate in an energy norm appropriate to (1.1).

On $\left[0, x_{M}\right]$ we use hat trial functions. Define a piecewise constant function $\tilde{a}$ which approximates $a(x)$ by

$$
\left.\tilde{a}_{i} \equiv \tilde{a}\right|_{\left(x_{i-1}, x_{i}\right]}=\left(a\left(x_{i-1}\right)+a\left(x_{i}\right)\right) / 2 \text { for } i=1, \ldots, N \text {. }
$$

Next, define a basis $\left\{\tilde{\phi}_{i}: i=M, \ldots, N-1\right\}$ for a space of approximate $L$-spline trial functions on $\left[x_{M}, 1\right]$ by

$$
\begin{aligned}
\tilde{L} \tilde{\phi}_{i} \equiv-\varepsilon \tilde{\phi}_{i}^{\prime \prime}+\tilde{a} \tilde{\phi}_{i}^{\prime}=0 & & \text { on }\left[x_{M}, 1\right]^{\wedge}, \\
\tilde{\phi}_{i}\left(x_{j}\right)=\delta_{i j} & & \text { for } j=M, \ldots, N,
\end{aligned}
$$

where means that mesh points are excluded. Our trial space $S$ now consists of hat trials on $\left[0, x_{M}\right]$ and $\widetilde{L}$-spline trials on $\left[x_{M}, 1\right]$; in particular, the basis function $\tilde{\phi}_{M}$ is a hybrid hat/ $\widetilde{L}$-spline.

We take our test space $T$ to be the span of $\left\{\psi_{i}: i=1, \ldots, N-1\right\}$, where each $\psi_{i}$ is the hat function satisfying $\psi_{i}\left(x_{j}\right)=\delta_{i j}$ for all $j$.

Lemma 4.1 (interpolation error in the $L^{\infty}$ norm). Let $u_{I} \in S$ interpolate to the solution $u$ of (1.1) at each node $x_{i}, i=0, \ldots, N$. Then for $x \in\left[x_{i-1}, x_{i}\right]$,

(i) $\left|\left(u-u_{I}\right)(x)\right| \leq C h_{i}^{2}$ if $1 \leq i \leq M$,

(ii) $\left|\left(u-u_{I}\right)(x)\right| \leq C h_{i}\left(1-e^{-\alpha h_{i} / \varepsilon}\right)$ if $M<i \leq N$.

Proof. Inequality (i) is a standard result using (3.1). To prove (ii), use a maximum principle/barrier function approach. See [17] for details.

The next result relates the $L^{1}$ and $L^{2}$ norms of the derivative of an $\widetilde{L}$-spline over each subinterval within the layer region.

Lemma 4.2. For each $w \in S$ and each $i \in\{M+1, \ldots, N\}$,

$$
\int_{x_{i-1}}^{x_{i}}\left|w^{\prime}(x)\right| d x \leq C\left(1-e^{-\alpha h_{i} / \varepsilon}\right)^{1 / 2} \varepsilon^{1 / 2}\left\{\int_{x_{i-1}}^{x_{i}}\left|w^{\prime}(x)\right|^{2} d x\right\}^{1 / 2} .
$$

Proof. Compute each side of the inequality in terms of $\left|w\left(x_{i}\right)-w\left(x_{i-1}\right)\right|$. See [17] for details. 
Definitions and notation. Let $(\cdot, \cdot)$ denote the usual $L^{2}[0,1]$ inner product. Let $(\cdot, \cdot)^{\wedge}$ denote that the integration is only over $[0,1] \backslash\left\{x_{0}, \ldots, x_{N}\right\}$.

Define $\|\cdot\|$ to be the usual $L^{2}[0,1]$ norm, and $\|\cdot\|_{d}$ to be its discrete analogue, viz.,

$$
\|w\|_{d} \equiv\left\{\sum_{i=1}^{N-1} \tilde{h}_{i} w^{2}\left(x_{i}\right)\right\}^{1 / 2},
$$

for all $w \in H_{0}^{1}(0,1)$. Then our energy norm is defined to be

$$
\||| w|| \equiv\left\{\varepsilon\left\|w^{\prime}\right\|^{2}+\|w\|_{d}^{2}\right\}^{1 / 2},
$$

for all $w \in H_{0}^{1}(0,1)$. Set

$$
B(v, w)=\left(\varepsilon v^{\prime}, w^{\prime}\right)+\left(a v^{\prime}, w\right)+(b v, w)
$$

and

$$
\widetilde{\boldsymbol{B}}(v, w)=\left(\varepsilon v^{\prime}, w^{\prime}\right)+\left(\tilde{a} v^{\prime}, w\right)+\sum_{i=1}^{N-1} \tilde{h}_{i}(b v w)\left(x_{i}\right)
$$

for all $v, w \in H_{0}^{1}(0,1)$.

We begin the analysis by showing that the bilinear form $\widetilde{B}(\cdot, \cdot)$ is coercive over $S \times T$.

Lemma 4.3. For each $v \in S$, let $v_{T} \in T$ interpolate to $v$ at each $x_{i}$. Then for $H$ sufficiently small (depending only on $b, a^{\prime}$, and $a^{\prime \prime}$ ),

$$
\widetilde{B}\left(v, v_{T}\right) \geq C_{1}\|\mid v\| \|^{2} \text {. }
$$

Proof. We have

$$
\begin{aligned}
\widetilde{B}\left(v, v_{T}\right)= & \widetilde{B}(v, v)+\widetilde{B}\left(v, v_{T}-v\right) \\
= & \varepsilon\left\|v^{\prime}\right\|^{2}+\sum_{i} \tilde{h}_{i}\left(b\left(x_{i}\right)-(1 / 2) a^{\prime}\left(x_{i}\right)+O\left(\tilde{h}_{i}\right)\right) v^{2}\left(x_{i}\right) \\
& +\left(-\varepsilon v^{\prime \prime}+\tilde{a} v^{\prime},\left(v_{T}-v\right)\right)^{-} \quad \begin{array}{r}
\text { (on integrating by parts and using } \\
\geq
\end{array} \\
& C_{1}\|v v\|^{2},
\end{aligned}
$$

for $H$ sufficiently small, where we have used $(1.1 \mathrm{~d}), v_{T}=v$ on $\left[0, x_{M}\right]$, and both (4.1) and $\left(v-v_{T}\right)\left(x_{i}\right)=0$ on $\left[x_{M}, 1\right]$.

Our computed solution $u^{h} \in S$ is obtained from the linear system of equations

$$
\widetilde{B}\left(u^{h}, \psi_{i}\right)=\tilde{h}_{i} f\left(x_{i}\right), \quad i=1, \ldots, N-1 .
$$

It follows from Lemma 4.3 that (4.3) has a unique solution.

Notation. We set $\rho=\alpha H / \varepsilon$. 
Proposition 4.4. For $H$ sufficiently small (independently of $\varepsilon$ ),

$$
\left\|\left|u_{I}-u^{h} \|\right| \leq C H+C H^{1 / 2}\left(1-e^{-\rho}\right)^{1 / 2} .\right.
$$

Proof. From Lemma 4.3, we have

$$
\begin{aligned}
C_{1}\left\|u_{I}-u^{h}\right\|^{2} & \leq \widetilde{B}\left(u_{I}-u^{h},\left(u_{I}-u^{h}\right)_{T}\right) \\
& =\widetilde{B}\left(u_{I}-u,\left(u_{I}-u^{h}\right)_{T}\right)+\widetilde{B}\left(u-u^{h},\left(u_{I}-u^{h}\right)_{T}\right) .
\end{aligned}
$$

We bound each of these $\widetilde{B}(\cdot, \cdot)$-terms separately. First,

$$
\begin{aligned}
\widetilde{B}\left(u_{I}-u,\left(u_{I}-u^{h}\right)_{T}\right) & =\left(u_{I}-u,-\varepsilon\left(u_{I}-u^{h}\right)_{T}^{\prime \prime}-\tilde{a}\left(u_{I}-u^{h}\right)_{T}^{\prime}\right)^{\wedge} \\
& =\left(u-u_{I}, \tilde{a}\left(u_{I}-u^{h}\right)_{T}^{\prime}\right),
\end{aligned}
$$

because $\left(u_{I}-u^{h}\right)_{T}$ is piecewise linear and $\left(u_{I}-u\right)\left(x_{i}\right)=0$ for each $i$. Hence,

$$
\begin{aligned}
\mid \widetilde{B}\left(u_{I}-\right. & \left.u,\left(u_{I}-u^{h}\right)_{T}\right) \mid \\
\leq & C \sum_{i=1}^{M} h_{i}^{2}\left|\left(u_{I}-u^{h}\right)\left(x_{i}\right)-\left(u_{I}-u^{h}\right)\left(x_{i-1}\right)\right| \\
& +C \sum_{i=M+1}^{N} h_{i}\left(1-e^{-\alpha h_{i} / \varepsilon}\right) \int_{x_{i-1}}^{x_{i}}\left|\left(u_{i}-u^{h}\right)^{\prime}(x)\right| d x \\
\leq & C \sum_{i=1}^{M}\left(\tilde{h}_{i}\right)^{2}\left|\left(u_{I}-u^{h}\right)\left(x_{i}\right)\right| \\
& +C \sum_{i=M+1}^{N} h_{i}\left(1-e^{-\alpha h_{i} / \varepsilon}\right)^{3 / 2} \varepsilon^{1 / 2}\left\{\int_{x_{i-1}}^{x_{i}}\left|\left(u_{I}-u^{h}\right)^{\prime}(x)\right|^{2} d x\right\}^{1 / 2} \\
\leq & C \sum_{i=1}^{M}\left(\tilde{h}_{i}\right)^{3}+\left(C_{1} / 8\right) \sum_{i=1}^{M} \tilde{h}_{i}\left|\left(u_{I}-u^{h}\right)\left(x_{i}\right)\right|^{2} \quad(\text { by Lemma 4.2) } \\
& +C\left(1-e^{-\rho}\right)^{3} \sum_{i=M+1}^{N-1}\left(\tilde{h}_{i}\right)^{2}+\left(C_{1} / 8\right) \varepsilon\left\|\left(u_{I}-u^{h}\right)^{\prime}\right\|^{2} \\
\leq & C H\left(1-e^{-\rho}\right)+C_{1} / 8 \mid\left\|u_{I}-u^{h}\right\| \|^{2} \quad\left(\text { since } \sum_{i=1}^{N-1} \tilde{h}_{i} \leq 1\right) .
\end{aligned}
$$


Returning to (4.4), we have

$$
\begin{aligned}
\widetilde{B}(u- & \left.u^{h},\left(u_{I}-u^{h}\right)_{T}\right)=\widetilde{B}\left(u,\left(u_{I}-u^{h}\right)_{T}\right)-\widetilde{B}\left(u^{h},\left(u_{I}-u^{h}\right)_{T}\right) \\
= & \widetilde{B}\left(u,\left(u_{I}-u^{h}\right)_{T}\right)-B\left(u,\left(u_{I}-u^{h}\right)_{T}\right)+\left(f,\left(u_{I}-u^{h}\right)_{T}\right) \\
& -\sum_{i=1}^{N-1} f\left(x_{i}\right) \tilde{h}_{i}\left(u_{I}-u^{h}\right)\left(x_{i}\right) \quad(\text { by }(1.1 \mathrm{a}) \text { and }(4.3)) \\
= & \left((\tilde{a}-a) u^{\prime},\left(u_{I}-u^{h}\right)_{T}\right)+\sum_{i} \tilde{h}_{i}\left(b u\left(u_{I}-u^{h}\right)\right)\left(x_{i}\right) \\
& -\left(b u,\left(u_{I}-u^{h}\right)_{T}\right)+\sum_{i}\left(u_{I}-u^{h}\right)\left(x_{i}\right)\left(f-f\left(x_{i}\right), \psi_{i}\right) .
\end{aligned}
$$

Set $\theta_{i}=\int_{x_{i-1}}^{x_{i+1}}\left|u^{\prime}(x)\right| d x$. Then, using bounds from Lemma 2.2, we have

$$
\theta_{i} \leq C \tilde{h}_{i}+C\left(e^{-\alpha\left(1-x_{i+1}\right) / \varepsilon}-e^{-\alpha\left(1-x_{i-1}\right) / \varepsilon}\right) .
$$

It follows [17] that

$$
\sum_{i} \theta_{i}^{2} \leq C H+C\left(1-e^{-\rho}\right)
$$

Thus,

$$
\begin{aligned}
\mid((\tilde{a}- & a) u^{\prime},\left(u_{I}-u^{h}\right)_{T}\left|\leq C \sum_{i=1}^{N-1} \tilde{h}_{i}\right|\left(u_{I}-u^{h}\right)\left(x_{i}\right) \mid \theta_{i} \\
& \leq \sum_{i} \tilde{h}_{i}\left[\left(C_{1} / 8\right)\left(u_{I}-u^{h}\right)^{2}\left(x_{i}\right)+C \theta_{i}^{2}\right] \quad \text { (using (2.3)) } \\
& \leq\left(C_{1} / 8\right)\left\|u_{I}-u^{h}\right\|_{d}^{2}+C H^{2}+C H\left(1-e^{-\rho}\right)
\end{aligned}
$$

As regards the terms involving $f$ from (4.6), we have

$$
\begin{aligned}
& \left|\sum_{i=1}^{N-1}\left(u_{I}-u^{h}\right)\left(x_{i}\right)\left(f-f\left(x_{i}\right), \psi_{i}\right)\right| \\
& \quad \leq C \sum_{i}\left(\tilde{h}_{i}\right)^{2}\left|\left(u_{I}-u^{h}\right)\left(x_{i}\right)\right| \\
& \quad \leq \sum_{i}\left\{C\left(\tilde{h}_{i}\right)^{3}+\left(C_{1} / 8\right) \tilde{h}_{i}\left(u_{I}-u^{h}\right)^{2}\left(x_{i}\right)\right\} \quad \text { (using (2.3)) } \\
& \quad \leq C H^{2}+\left(C_{1} / 8\right)\left\|u_{I}-u^{h}\right\|_{d}^{2} .
\end{aligned}
$$

Taking the remaining terms in (4.6), we can essentially imitate the previous calculations to obtain

$$
\begin{aligned}
& \left|\sum_{i=1}^{N-1} \tilde{h}_{i}\left(b u\left(u_{I}-u^{h}\right)\right)\left(x_{i}\right)-\left(b u,\left(u_{I}-u^{h}\right)_{T}\right)\right| \\
& \quad \leq\left(C_{1} / 8\right)\left\|u_{I}-u^{h}\right\|_{d}^{2}+C H^{2}+C H\left(1-e^{-\rho}\right) .
\end{aligned}
$$

Now substituting (4.7), (4.8), and (4.9) into (4.6), we obtain

$$
\left|\widetilde{B}\left(u-u^{h},\left(u_{I}-u^{h}\right)_{T}\right)\right| \leq\left(3 C_{1} / 8\right) \mid\left\|u_{I}-u^{h}\right\|^{2}+C H^{2}+C H\left(1-e^{-\rho}\right) .
$$


Combining this inequality with (4.4) and (4.5) then completes the proof of Proposition 4.4 .

We now need to estimate $\left\|u-u_{I}\right\|$.

Proposition 4.5 (interpolation error in the energy norm). For all meshes we have

$$
\left\|u-u_{I}\right\| \|^{2} \leq C H\left(H+\left(1-e^{-\rho}\right) \varepsilon \ln (1 / \varepsilon)\right) .
$$

Proof. Integrating by parts, we have

$$
\begin{aligned}
B\left(u-u_{I}, u-u_{I}\right) & =\left(\varepsilon\left(u-u_{I}\right)^{\prime},\left(u-u_{I}\right)^{\prime}\right)+\left(b-a^{\prime} / 2,\left(u-u_{I}\right)^{2}\right) \\
& \left.\geq\left\|u-u_{I}\right\|^{2} \quad \text { (using (1.1d) and }\left\|u-u_{I}\right\|_{d}=0\right) .
\end{aligned}
$$

On the other hand,

$$
\begin{aligned}
B\left(u-u_{I}, u-u_{I}\right)= & \left(f-b u_{I}, u-u_{I}\right)-\int_{0}^{x_{M}} a u_{I}^{\prime}\left(u-u_{I}\right) d x \\
& +\int_{x_{M}}^{1}(\tilde{a}-a) u_{I}^{\prime}\left(u-u_{I}\right) d x
\end{aligned}
$$

by (1.1a) and our choice of trial functions. Here,

$$
\begin{aligned}
\mid\left(f-b u_{I},\right. & \left.u-u_{I}\right)\left|\leq C \int_{0}^{x_{M}}\right| u-u_{I}\left|d x+C \int_{x_{M}}^{1}\right| u-u_{I} \mid d x \\
& \leq C H^{2}+C\left(1-x_{M}\right) H\left(1-e^{-\rho}\right) \quad(\text { by Lemma 4.1) } \\
& \leq C H^{2}+C H\left(1-e^{-\rho}\right)(H+\varepsilon \ln (1 / \varepsilon)) \quad(\text { by choice of } M) \\
& \leq C H^{2}+C H\left(1-e^{-\rho}\right) \varepsilon \ln (1 / \varepsilon) .
\end{aligned}
$$

Also,

$$
\begin{gathered}
\left|\int_{0}^{x_{M}} a u_{I}^{\prime}\left(u-u_{I}\right) d x+\int_{x_{M}}^{1}(\tilde{a}-a) u_{I}^{\prime}\left(u-u_{I}\right) d x\right| \\
\quad \leq C \sum_{i=1}^{N} h_{i}^{2} \int_{x_{i-1}}^{x_{i}}\left|u_{I}^{\prime}(x)\right| d x \quad \text { (by Lemma 4.1) } \\
\quad \leq C \sum_{i=1}^{N} h_{i}^{2}\left|u\left(x_{i}\right)-u\left(x_{i-1}\right)\right| \leq C H^{2}
\end{gathered}
$$

since $\int_{0}^{1}\left|u^{\prime}(x)\right| d x \leq C$ from (2.2).

Combining (4.10)-(4.13), we get the desired result.

Remark 4.6. Since $1-e^{-\rho}<\rho$ for all $\rho>0$, this bound essentially states that $\left\|u-u_{I}\right\| \mid$ is almost $O(H)$.

We can now easily derive the main result of this section. 
Theorem 4.7 (energy norm error estimate). For $H$ sufficiently small (independently of $\varepsilon$ ), and $u^{h}$ defined by (4.3),

$$
\left\|u-u^{h}\right\| \leq C H+C H^{1 / 2}\left(1-e^{-\rho}\right)^{1 / 2} .
$$

Proof. Combine Propositions 4.4 and 4.5.

Remark 4.8. Theorem 4.7 trivially implies that, uniformly in $\varepsilon,\left\|u-u^{h}\right\|_{d} \leq$ $C H^{1 / 2}$. It is possible to show that, on a uniform mesh, $\left\|u-u^{h}\right\|_{d} \leq C H$.

\section{5. $\bar{L}^{*}$-SPLINE TEST FUNCTIONS IN THE LAYER REGION}

We now consider a different Petrov-Galerkin finite element method for solving (1.1). This method is closely related to one used in Stynes [14]. However, the analysis in [14] is finite difference in nature, whereas the argument below uses only finite element techniques. We prove an $L^{2}$ error estimate of order $H$ for the method. This estimate is obtained directly, without use of any energy norm error estimate.

Assume that the mesh is arbitrarily graded on $\left[x_{M-1}, 1\right]$, i.e., $h_{M} \geq h_{M+1} \geq$ $\cdots \geq h_{N}$. This is not a practical restriction, as it would be quite unusual to coarsen the mesh as one moves into the boundary layer. The mesh on $\left[0, x_{M-1}\right]$ is still unrestricted. With a view to proving an $O(H)$ error bound in the $L^{2}$ norm on such a general mesh, we replace the approximation $\tilde{a}$ by a modified piecewise constant approximation $\bar{a}$ in order to obtain the coercivity result of Lemma 5.1. For the definition of $\bar{a}$, see [14]. Regarding $\bar{a}$ as a quadrature rule which enables evaluation of those integrals arising in the finite element method, it has the property of automatically varying with the local-cell Reynolds number in such a way as to ensure stability of the method. In particular, on intervals $\left[x_{i-1}, x_{i}\right]$, where $h_{i}<<\varepsilon$, we have $\bar{a}_{i} \approx \tilde{a}_{i}$, and on intervals where $h_{i}>>\varepsilon$, we have $\bar{a}_{i} \approx a\left(x_{i-1}\right)$.

Take the trial space $S$ to be the span of $\left\{\phi_{i}: i=1, \ldots, N-1\right\}$, where each $\phi_{i}$ is the hat function satisfying $\phi_{i}\left(x_{j}\right)=\delta_{i j}$ for all $j$.

In our test space $T$ we use hat functions on $\left[0, x_{M}\right]$. Our approximate $L^{*}$-spline test functions are defined as follows: for $i=M, \ldots, N-1$, let $\bar{\psi}_{i}$ satisfy

$$
\begin{gathered}
\bar{L}^{*} \bar{\psi}_{i} \equiv-\varepsilon \bar{\psi}_{i}^{\prime \prime}-\bar{a} \bar{\psi}_{i}^{\prime}=0 \quad \text { on }\left[x_{M}, 1\right]^{-}, \\
\bar{\psi}_{i}\left(x_{j}\right)=\delta_{i j}, \quad j=M, \ldots, N .
\end{gathered}
$$

Our test space $T$ is the span of $\left\{\bar{\psi}_{i}: i=1, \ldots, N-1\right\}$, where for $i=$ $1, \ldots, M-1, \bar{\psi}_{i}$ is a hat function centered at $x_{i}$, and $\bar{\psi}_{M}$ is a hybrid hat $/ \bar{L}^{*}$-spline.

We also define a set of functions $\left\{\tilde{\psi}_{i}: i=1, \ldots, N-1\right\}$ analogous to the $\left\{\bar{\psi}_{i}\right\}$. We take each $\left\{\tilde{\psi}_{i}\right\}$ to satisfy $\widetilde{L}^{*} \tilde{\psi}_{i}=0$ on $\left[x_{M}, 1\right]^{\wedge}$; in all other respects, $\tilde{\psi}_{i}$ is defined as $\bar{\psi}_{i}$ was defined.

Set

$$
\|w\|_{d^{\prime}}=\left\{\sum_{i=1}^{N-1}\left(1, \tilde{\psi}_{i}\right) w^{2}\left(x_{i}\right)\right\}^{1 / 2}
$$


for all $w \in H_{0}^{1}(0,1)$. On $H_{0}^{1}(0,1)$, this norm is equivalent to the discrete $L^{2}$ norm defined in the last section (see [17]).

Set

$$
\bar{B}(v, w)=\left(\varepsilon v^{\prime}, w^{\prime}\right)+\left(\bar{a} v^{\prime}, w\right)+\sum_{i=1}^{N-1}\left(1, \tilde{\psi}_{i}\right)(b v w)\left(x_{i}\right)
$$

for all $v, w \in H_{0}^{1}(0,1)$.

Lemma 5.1. For each $v \in S$, let $v_{T} \in T$ interpolate to $v$ at each $x_{i}$. Then for $H$ sufficiently small (depending only on $b, a^{\prime}$, and $a^{\prime \prime}$ ),

Proof. See [17].

$$
\bar{B}\left(v, \dot{v}_{T}\right) \geq C_{1}\left\|v_{T}\right\|^{2} \text {. }
$$

Our computed solution $u^{h} \in S$ is now obtained from the linear system of equations

$$
\bar{B}\left(u^{h}, \bar{\psi}_{i}\right)=\left(1, \tilde{\psi}_{i}\right) f\left(x_{i}\right), \quad i=1, \ldots, N-1 .
$$

To obtain an estimate for $\left\|u-u^{h}\right\|_{d^{\prime}}$, we employ a duality argument. Assume that $\left\|u_{I}-u^{h}\right\|_{d^{\prime}} \neq 0$, as otherwise we are done. Define $w \in T$ by

$$
\bar{B}\left(\phi_{j}, w\right)=\frac{\sum_{i=1}^{N-1}\left(1, \tilde{\psi}_{i}\right)\left(u_{I}-u^{h}\right)\left(x_{i}\right)\left(\phi_{j}\right)\left(x_{i}\right)}{\left\|u_{I}-u^{h}\right\|_{d^{\prime}}}, \quad j=1, \ldots, N-1 .
$$

Now, letting $u_{I} \in S$ interpolate to $u$ at each node,

$$
\begin{aligned}
\left\|u-u^{h}\right\|_{d^{\prime}} & =\left\|u_{I}-u^{h}\right\|_{d^{\prime}}=\bar{B}\left(u_{I}-u^{h}, w\right) \quad(\text { from }(5.2)) \\
& =\bar{B}\left(u_{I}-u, w\right)+\bar{B}\left(u-u^{h}, w\right) .
\end{aligned}
$$

Theorem 5.2 (discrete $L^{2}$ norm error estimate). For $H$ sufficiently small (indepently of $\varepsilon$ ), and $u^{h}$ defined by (5.1),

$$
\left\|u-u^{h}\right\|_{d} \leq C H \text {. }
$$

Proof. The two right-hand side terms of (5.3) can be bounded separately, using arguments similar to those of $\S 4$. See [17] for details.

\section{Simultaneous ENERgy AND $L^{2}$ NORM ERROR Estimates}

Let the trial space $S$ be as in $\S 4$ (so $S$ consists of $\widetilde{L}$-splines in the layer region), and let the test space $T$ be as in $\S 5$ (so $T$ consists of $\bar{L}^{*}$-splines in the layer region). Let the mesh be arbitrarily graded in the layer region as in $\S 5$. We use the bilinear form $\bar{B}(\cdot, \cdot)$ of $\S 5$.

Define our computed solution $u^{h} \in S$ by

$$
\bar{B}\left(u^{h}, \bar{\psi}_{i}\right)=\left(1, \tilde{\psi}_{i}\right) f\left(x_{i}\right), \quad i=1, \ldots, N-1 .
$$

The following theorem improves the main result of $\S 4$; it also includes a result similar to that of $\S 5$. 
Theorem 6.1 [17]. For $H$ sufficiently small (independently of $\varepsilon$ ), and $u^{h}$ defined by $(6.1)$,

$$
\left\|u-u^{h}\right\| \mid \leq C H^{1 / 2}\left(H+\left(1-e^{-\rho}\right) \varepsilon \ln (1 / \varepsilon)\right)^{1 / 2}
$$

and

$$
\left\|u-u^{h}\right\|_{d} \leq C H .
$$

Corollary 6.2 [17]. For $H$ sufficiently small (independently of $\varepsilon$ ), and $u^{h} d e$ fined by (6.1),

$$
\left\|u-u^{h}\right\| \leq C H
$$

\section{ACKNOWLEDGEMENT}

We wish to thank the referee for the thorough report on the initial version of this paper.

\section{BIBLIOGRAPHY}

1. O. Axelsson, Stability and error estimates of Galerkin finite element approximations for convection-diffusion equations, IMA J. Numer. Anal. 1 (1981), 329-345.

2. A. E. Berger, J. M. Solomon, and M. Ciment, An analysis of a uniformly accurate difference method for a singular perturbation problem, Math. Comp. 37 (1981), 79-94.

3. K. V. Emelyanov, Difference schemes for singularly perturbed boundary value problems, BAIL IV (S. K. Godunov, J. J. H. Miller, and V. A. Novikov, eds.), Boole Press, Dublin, 1986, pp. 51-60.

4. E. C. Gartland, Jr., An analysis of a uniformly convergent finite difference/finite element scheme for a model singular-perturbation problem, Math. Comp. 51 (1988), 93-106.

5. __ Graded-mesh difference schemes for singularly perturbed two-point boundary value problems, Math. Comp. 51 (1988), 631-657.

6. P. P. N. de Groen, $A$ finite element method with a large mesh-width for a stiff two-point boundary value problem, J. Comput. Appl. Math. 7 (1981), 3-15.

7. A. F. Hegarty, E. O'Riordan, and M. Stynes, A comparison of uniformly convergent difference schemes for two-dimensional convection-diffusion problems (in preparation).

8. A. M. Il ' in, Differencing scheme for a differential equation with a small parameter affecting the highest derivative, Mat. Zametki 6 (1969), 237-248; English transl. in Math. Notes 6 596-602.

9. R. B. Kellogg and A. Tsan, Analysis of some difference approximations for a singular perturbation problem without turning points, Math. Comp. 32 (1978), 1025-1039.

10. K. W. Morton, Galerkin finite element methods and their generalizations, The State of the Art in Numerical Analysis (A. Iserles and M. J. D. Powell, eds.), Clarendon Press, Oxford, 1987, pp. 645-680.

11. K. W. Morton and B. W. Scotney, Petrov-Galerkin methods and diffusion-convection problems in 2D, The Mathematics of Finite Elements and Applications V (MAFELAP 1984) (J. R. Whiteman, ed.), Academic Press, London, 1985, pp. 343-366.

12. K. Niederdrenk and H. Yserentant, Die gleichmässige Stabilität singulär gestörter diskreter und kontinuierlicher Randwertprobleme, Numer. Math. 41 (1983), 223-253.

13. E. O'Riordan and M. Stynes, A globally uniformly convergent finite element method for a singularly perturbed elliptic problem in two dimensions, Math. Comp. 57 (1991) (to appear).

14. M. Stynes, An adaptive uniformly convergent numerical method for a semilinear singular perturbation problem, SIAM J. Numer. Anal. 26 (1989), 442-455. 
15. M. Stynes and E. O'Riordan, A finite element method for a singularly perturbed boundary value problem, Numer. Math. 50 (1986), 1-15.

16. _ Finite element methods for elliptic convection-diffusion problems, BAIL V, Proc 5th Internat. Conf. on Boundary and Interior Layers (B. Guo, J. J. H. Miller, and Z. Shi, eds.), Boole Press, Dublin, 1988, pp. 65-76.

17. __ An analysis of a two-point boundary value problem with a boundary layer, using only finite element techniques, Technical Report, Department of Mathematics, University College, Cork, October 1989.

18. W. G. Szymczak and I. Babuška, Adaptivity and error estimation for the finite element method applied to convection diffusion problems, SIAM J. Numer. Anal. 21 (1984), 910954.

19. M. Van Veldhuizen, Higher order schemes of positive type for singular perturbation problems, Numerical Analysis of Singular Perturbation Problems (P. W. Hemker and J. J. H. Miller, eds.), Academic Press, New York, 1979, pp. 361-383.

Department of Mathematics, University College, Cork, Ireland

E-mail address: stynes@math-cs.kent.edu

Department of Mathematics, Regional Technical College, Dundalk, Ireland

Current address, M. Stynes: Department of Mathematical Sciences, Kent State University, Kent, Ohio 44242-0001 